Case Report

\title{
Autologous Bone-Marrow-Derived-Mononuclear-Cells-Enriched Fat Transplantation in Breast Augmentation: Evaluation of Clinical Outcomes and Aesthetic Results in a 30-Year-Old Female
}

\author{
Dmitry Bulgin, ${ }^{1}$ Erik Vrabic, ${ }^{1,2}$ and Enes Hodzic ${ }^{1}$ \\ ${ }^{1}$ Polyclinic "ME-DENT," 18 Istarska, 52210 Rovinj, Croatia \\ ${ }^{2}$ Department of Plastic and Reconstructive Surgery, University Clinical Centre (UCC), 5 Ljubljanska, 2000 Maribor, Slovenia
}

Correspondence should be addressed to Dmitry Bulgin; molmed1999@yahoo.com

Received 6 June 2013; Accepted 24 July 2013

Academic Editors: J. M. Bernal, K. Honma, G. Lal, C. Schmitz, and M. Zafrakas

Copyright (C) 2013 Dmitry Bulgin et al. This is an open access article distributed under the Creative Commons Attribution License, which permits unrestricted use, distribution, and reproduction in any medium, provided the original work is properly cited.

\begin{abstract}
Autologous fat transfer (lipofilling) is becoming an invaluable tool for breast augmentation as well as for breast reconstruction. Autologous lipofilling has several advantages, including biocompatibility, versatility, natural appearance, and low donor site morbidity. The main limitation is unpredictable fat graft resorption, which ranges from $25 \%$ to $80 \%$, probably as a result of ischaemia and lack of neoangiogenesis. To obviate these disadvantages, several studies have searched for new ways of increasing the viability of the transplanted fat tissue. One promising approach is to enrich the fat graft with autologous bone-marrow-derived mononuclear cells (BMMNCs) before transplantation. BMMNCs produce many angiogenic and antiapoptotic growth factors, and their secretion is significantly enhanced by hypoxia. All of these mechanisms of actions could be beneficial for the stimulation of angiogenesis in ischemic tissues by BMMNCs administration. In our aesthetic surgery practice, we use fat transplantation enriched with BMMNCs, which caused a significant improvement in survival of fat grafts, compared with that of traditional lipofilling. Our experience with freshly isolated autologous fat enriched with BMMNCs for breast augmentation procedures is presented. The concept of this surgical and tissue handling technique is based on ability of BMMNCs to stimulate blood vessel growth.
\end{abstract}

\section{Introduction}

After numerous experiments since 1893 [1, 2], autologous fat transplantation has become a well-established and frequently applied method of soft tissue augmentation for both cosmetic and reconstructive purposes. Tissue augmentation by fat grafting does have several advantages, and many promising results of autologous fat grafts have been published recently [3-8]. Autologous fat is an excellent and extremely promising soft-tissue filler, given its abundance and ease of harvest [9-13]. Selecting suitable indications and correct surgical techniques, low complication rate, and positive results make autologous fat grafting an ideal method for breast augmentation $[14,15]$.

Published clinical experience in over 2,000 patients who received autologous fat grafts showed no evidence of increased risk of development, metastasis, or recurrence of breast cancer [16-19]. The most significant drawback to autologous fat grafting remains its largely unpredictable rate of resorption, low rate of graft survival due to partial necrosis, and scattered microcalcifications, followed by dispersed radiolucent oil cysts [20-22]. Numerous in vitro and in vivo studies on fat graft viability have recently been done. According to results of these studies, the use of bone-marrow-derived cells is a novel approach to survival of fat grafts [23-25].

The autologous application of BMMNCs which are not expanded ex vivo has medicolegal advantages for clinical use [26]. BMMNCs as a huge source of bone-marrow-derived mesenchymal stem cells (BMMSCs) [27-31] represent a potential key component in the field of regenerative medicine and tissue engineering [32-34]. BMMSCs are multipotent and secrete many kinds of growth factors to regenerate tissues. During the past decade, numerous studies have provided preclinical and clinical data on the safety and efficacy 


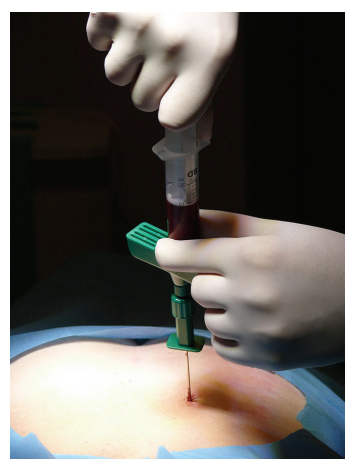

(a)

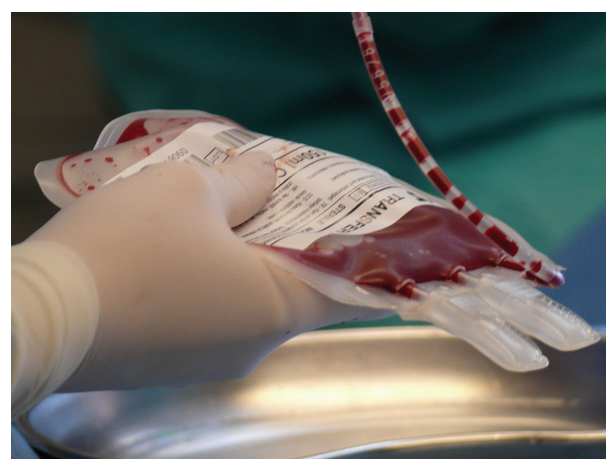

(b)

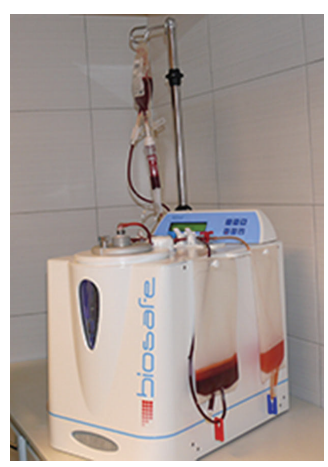

(c)

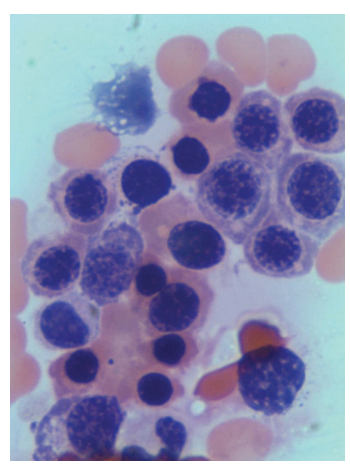

(d)

Figure 1: Bone-marrow-derived mononuclear cells preparation: (a) the bone marrow harvesting from posterior iliac crest, (b) collection of bone marrow in plastic bag, (c) the bone marrow processing by using Cell Separation System SEPAX S-100, and (d) qualitative assessment of BMMNCs population by haematoxylin and eosin cytological staining $(\times 400)$.

of BMMSCs, supporting the use of these cells in a wide range of clinical applications such as plastic surgery, cardiac surgery, orthopedic surgery, and oral and maxillofacial surgery [35-40]. BMMNCs stimulated angiogenesis as well as maturation of the newly formed blood vessels in vivo. These mechanisms of actions could be beneficial for the stimulation of angiogenesis in ischemic tissues by BMMNCs administration [41]. According to these characteristics, we suggest that the BMMNCs-enriched lipografts can produce aesthetically acceptable results without the need for repeating treatment sessions, which are necessary with autologous fat transplantation.

\section{Materials and Methods}

2.1. Patient. The patient is a 30-year-old female who requested bilateral breast augmentation. The patient signed a detailed informed consent form of the procedure and possible complications.

The patient has been carefully monitored with preoperative and serial postoperative ultrasonograms (preoperatively, at day 1; postoperatively, after 2 weeks, 4 weeks, 3 months, 6 months, and 12 months).

\subsection{Bone-Marrow-Derived Mononuclear Cells Preparation.} Autologous bone marrow from the patient was used as a source for BMMNCs. Under general anesthesia, $70.0 \mathrm{~mL}$ of the bone marrow was harvested from posterior iliac crest. BMMNCs were separated according to generic volume reduction protocol by using Cell Separation System SEPAX S100/a table top centrifuge system (Biosafe Group SA, Eysins, Switzerland). The SEPAX S-100 is a cell processing system that uses a rotating syringe technology that provides both separation through rotation of the syringe chamber (centrifugation) and component transfer through displacement of the syringe piston. The SEPAX S-100 system allows the automated processing of cell components in a functionally closed and sterile environment. The SEPAX S-100 provides centrifugal and axial displacement drive to the chamber on the singleuse separation kit, as well as drive to the directional valves.
The cell separation process is permanently monitored by an optical sensor, fully automated, and completed within 15 to 20 minutes and required a minimum of (nonspecialized) operator intervention. The generic volume reduction protocol uses a single sedimentation step with centrifugal force of $960 \times \mathrm{g}$ and concentrates the final cell product. After processing of the bone marrow, the final BMMNCs product was suspended in $15 \mathrm{~mL}$ autologous plasma. Quantitative assessment of cell population by Sysmex KX-21N cell counter (Sysmex Corp., Kobe, Japan), Trypan Blue exclusion test of cell viability, and in vitro haematoxylin and eosin cytological staining was performed to confirm viability and composition of BMMNCs (Figure 1).

\subsection{Purified Fat and BMMNCs Mixture Preparation and} Breast Augmentation Procedure. After BMMNCs preparation during the same anesthetic event, the patient was admitted for the next surgical procedures which included tumescent syringe liposuction [42] by the Coleman technique, which is based on manual aspiration [43, 44]. Fat was harvested mainly from the abdomen, thighs, and flanks. The harvested fat was transferred into a rigorously closed system Cytori PureGraft 250/PURE System (Cytori Therapeutics, Inc., San Diego, CA, USA). The PureGraft 250 System is indicated for autologous fat transfers. The PureGraft System allows the user to prepare fat grafts within the sterile field in less than 15 minutes. PureGraft selectively washes the graft, drains the tumescent fluid, and removes free lipid and debris. After processing the obtained purified fat and $15.0 \mathrm{~mL}$ of BMMNCs product were mixed in the same closed sytem (PureGraft 250 System). The BMMNCs and purified fat mixture was transferred to $10 \mathrm{~mL}$ syringes for injection directly into the breast by using micro droplet injection device Celbrush (Cytori Therapeutics, Inc., San Diego, USA) (Figure 2).

Cytori's Celbrush is a stainless steel device intended for use in the delivery of an autologous fat graft. The thumbbrush design gives the Celbrush a mechanical advantage that minimizes the buildup of pressure and provides superior tactile feedback during tissue dispersion. The $10 \mathrm{~mL}$ Celbrush 


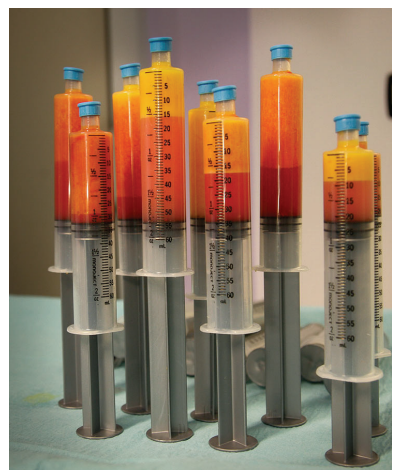

(a)

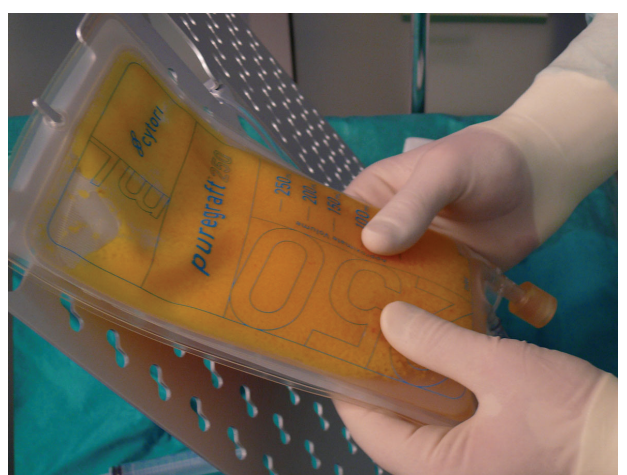

(b)

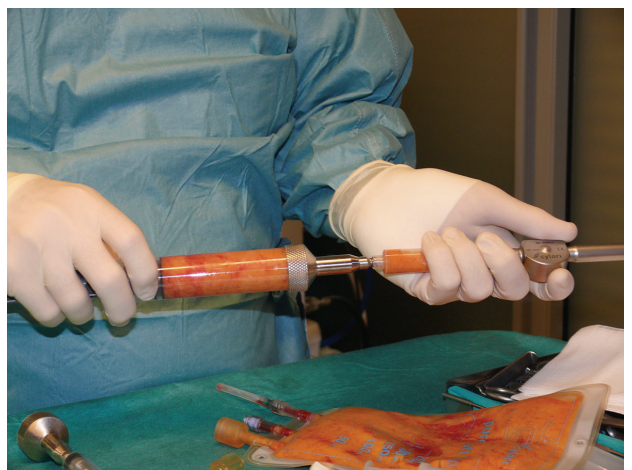

(c)

FIgURE 2: Purified fat and BMMNCs mixture preparation: (a) the harvested fat after tumescent syringe liposuction, (b) the purified fat in Cytori PureGraft 250/PURE System, and (c) the BMMNCs and purified fat mixture transferring to $10 \mathrm{~mL}$ syringe.

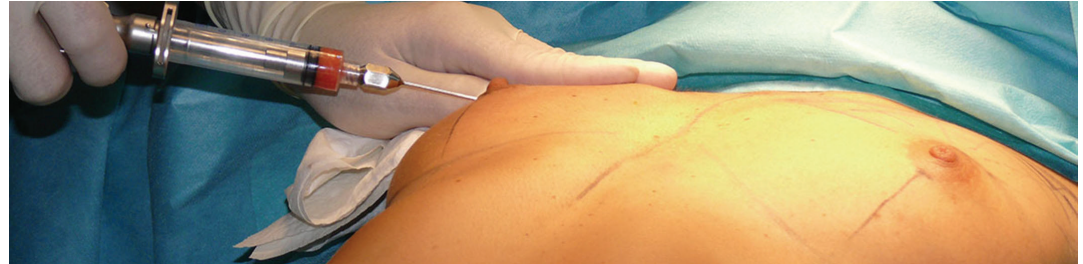

(a)

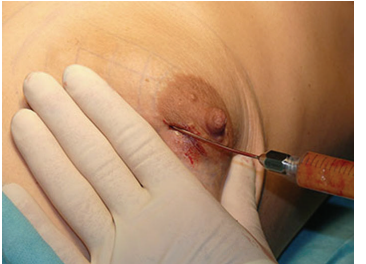

(b)

FIGURE 3: Purified fat and BMMNCs mixture reimplantation: (a) and (b) breast augmentation procedure (bicompartmental grafting).

is designed to deliver approximately $0.50 \mathrm{~mL}$ of tissue for each full brush of the operator's thumb. Minimally manipulated fat combined with BMMNCs was reimplanted strictly in two planes only: into the retroglandular and prefascial space and into the superficial subcutaneous plane of the upper pole of the breast (bicompartmental grafting) (Figure 3).

Any intraparenchymal placement was carefully avoided. Total grafted fat volume was $185.0 \mathrm{~mL}$ per breast. Average operation time was $3.5 \mathrm{~h}$. Postoperative follow-up was uneventful and no complications were observed.

\section{Results}

The patient was satisfied with the soft and natural-appearing augmentation. The breast mounds were soft with no subcutaneous induration and visible injection scars. Postoperative atrophy of injected fat was minimal and did not change substantially after 12 months (Figure 4).

The patient demonstrated improvement in circumferential breast measurement (BRM) from baseline state, and breast measurements were stable by 3 months after surgery. The BRM 12 months after surgery had increased $5.5 \mathrm{~cm}$ from preoperative measurements. Breast ultrasound showed no evidence of cyst formation or microcalcification.

\section{Discussion}

During the past decade, numerous studies have provided preclinical data on the safety and efficacy of BMMNCs, supporting the use of these cells in future clinical applications. Various clinical outcomes have shown the regenerative capability of BMMNCs in subspecialties of medical fields such as plastic surgery, orthopedic surgery, oral and maxillofacial surgery, and cardiac surgery $[35,39,40,45]$. These preliminary results suggest that BMMNCs-assisted lipotransfer could be effective and safe for soft tissue augmentation and superior to conventional lipoinjection. BMMNCs are an alternative cell source for obtaining mesenchymal stem cells (MSCs). The idea of using BMMNCs autografts is based on the assumption that MSCs among the mononuclear cells, which are present in only relatively small numbers in bone marrow aspirates, can be easily separated ex vivo from the rest of the harvested cells, concentrated in small volume, and immediately implanted into the patient's injured tissue, where the microenvironment will trigger their replication (i.e., cloning) and differentiation into specialized cells. MSCs produce many angiogenic and antiapoptotic growth factors, and their secretion is significantly enhanced by hypoxia [46]. MSCs enhance blood vessel growth not only by production of paracrine-acting factors but also by promoting the endothelial cells differentiation [47]. All of these mechanisms of 


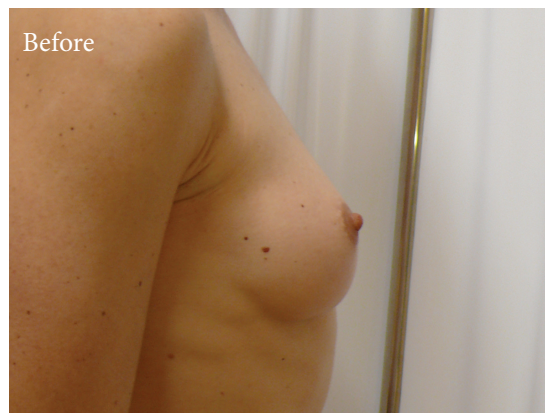

(A)

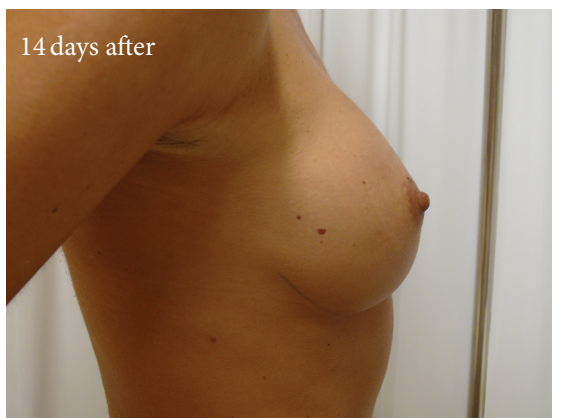

(A)

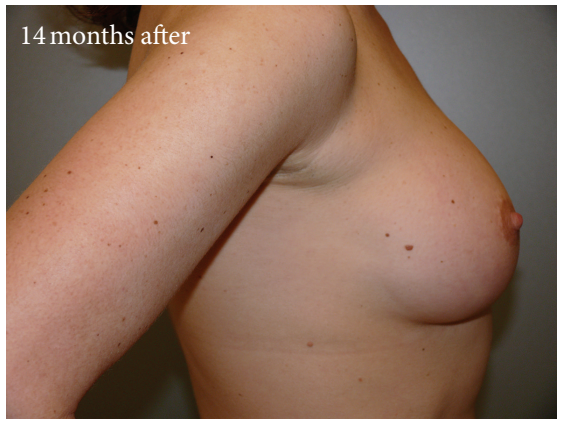

(A)

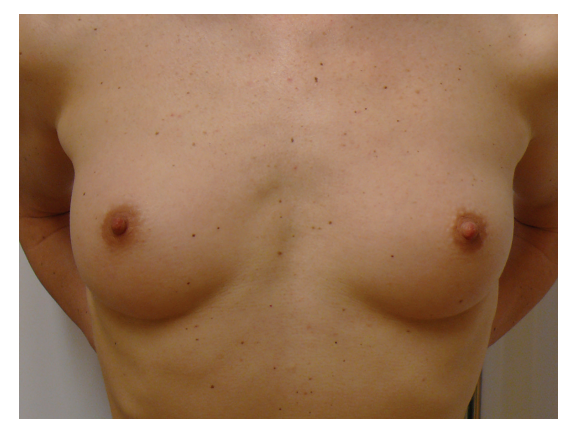

(B)

(a)

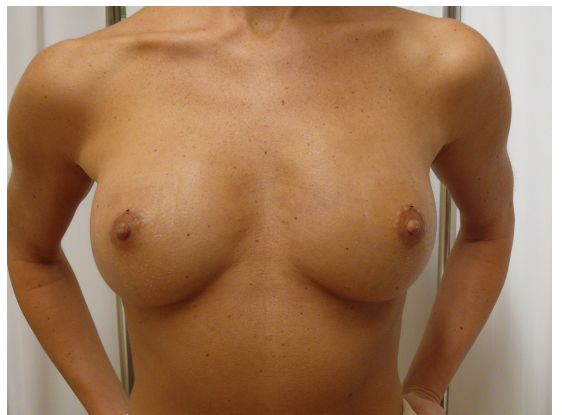

(B)

(b)

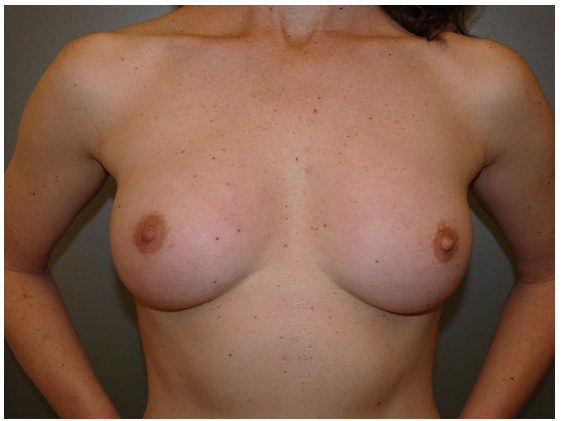

(B)

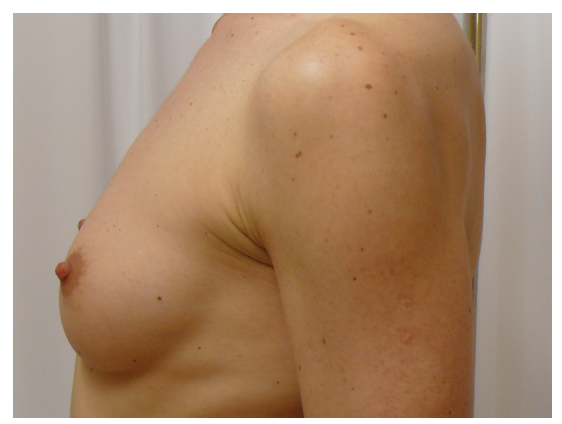

(C)

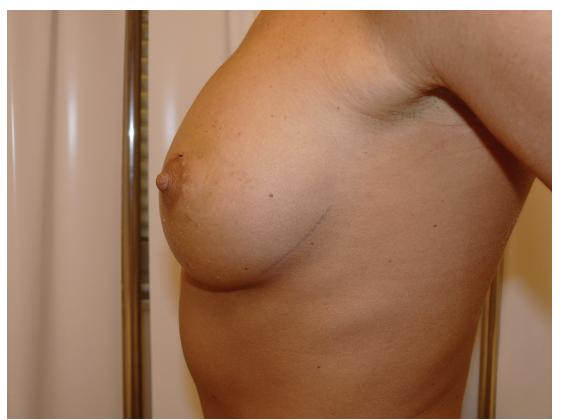

(C)

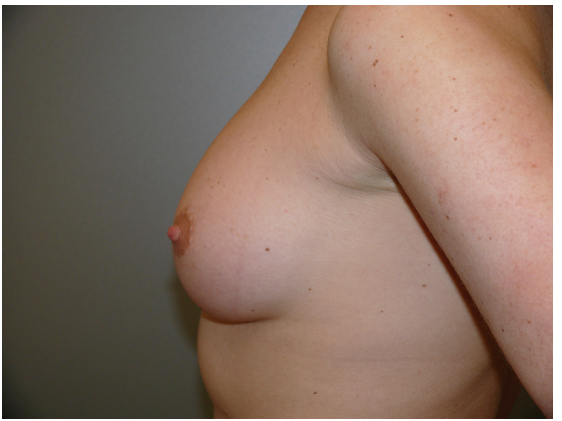

(C)

(c)

FIGURE 4: Clinical outcomes and aesthetic results after BMMNCs-enriched lipograft for primary bilateral breast augmentation: preoperative views (top), and postoperative views at 14 days (middle), postoperative views at 12 months (bottom); (A) right view, (B) front view, and (C) left view.

actions could be beneficial for the stimulation of angiogenesis in transplanted fat by BMMNCs administration. Although an optimal method of cells-assisted autologous fat grafting for primary breast augmentation should be standardized, further strong-evidence-based studies are necessary to confirm the findings of this approach $[48,49]$.

Breast ultrasound, an accurate and simple imaging technique, plays an important role in follow-up for temporal changes of fat nodules after autologous fat injection. Furthermore, breast ultrasound may avoid unnecessary biopsies [50].

\section{Conclusions}

It was observed that autologous fat combined with freshly isolated BMMNCs possessed excellent handling characteristics, with no adverse tissue reaction and infection. BMMNCs appear to be an ideal population of stem cells for practical regenerative medicine, given that they are plentiful, of autologous tissue origin and thus nonimmunogenic, and more easily available because of minimal ethical considerations. 
The advantages of this method for clinical use in primary breast augmentation are as follows:

(i) the cells do not need to be expanded in vitro; they preserve their angiogenic potential to form new blood vessels and promote the proper graft survival;

(ii) the aspirated fat is used as a living scaffold.

Our clinical outcomes showed that the procedure is safe and effective, providing improvement after a single treatment. Further long-term studies are necessary to confirm the favorable results seen in this study.

\section{Acknowledgments}

The authors would like to thank Dr. Sadanori Akita, Dr. Hiroshi Yoshimoto, and Mr. Robert Sekac for their contribution to the case report.

\section{References}

[1] G. A. Neuber, "Verhandlungen der Deutschen Gesellschaft für Chirurgie," p. 66, 1893. (German).

[2] V. Czerny, "Plastic replacement of the breast with a lipoma," Chirurgie Kong Verhandlungen, no. 2, p. 216, 1895.

[3] D. A. Hudson, E. V. Lambert, and C. E. Bloch, "Site selection for fat autotransplantation: some observations," Aesthetic Plastic Surgery, vol. 14, no. 3, pp. 195-197, 1990.

[4] I. Sarfati, T. Ihrai, G. Kaufman, C. Nos, and K. B. Clough, "Adipose-tissue grafting to the post-mastectomy irradiated chest wall: preparing the ground for implant reconstruction," Journal of Plastic, Reconstructive and Aesthetic Surgery, vol. 64, no. 9, pp. 1161-1166, 2011.

[5] A. A. Lim, K. Fan, K. A. Allam et al., "Autologous fat transplantation in the craniofacial patient: the UCLA experience," The Journal of Craniofacial Surgery, vol. 23, no. 4, pp. 1061-1066, 2012.

[6] A. R. Scorza, L. R. Scorza, A. Troccola, D. M. Micci, R. Rauso, and G. Curinga, "Autologous fat transfer for face rejuvenation with tumescent technique fat harvesting and saline washing: a report of 215 cases," Dermatology, vol. 224, no. 3, pp. 244-250, 2012.

[7] A. Losken, X. A. Pinell, K. Sikoro, M. V. Yezhelyev, E. Anderson, and G. W. Carlson, "Autologous fat grafting in secondary breast reconstruction," Annals of Plastic Surgery, vol. 66, no. 5, pp. 518$522,2011$.

[8] H. Mizuno and H. Hyakusoku, "Fat grafting to the breast and adipose-derived stem cells: recent scientific consensus and controversy," Aesthetic Surgery Journal, vol. 30, no. 3, pp. 381-387, 2010.

[9] J. Smahel, "Adipose issue in plastic surgery," Annals of Plastic Surgery, vol. 16, no. 5, pp. 444-453, 1986.

[10] M. Bircoll and B. H. Novack, "Autologous fat transplantation employing liposuction techniques," Annals of Plastic Surgery, vol. 18 , no. 4, pp. 327-329, 1987.

[11] L. P. Bucky and I. Percec, "The science of autologous fat grafting: views on current and future approaches to neoadipogenesis," Aesthetic Surgery Journal, vol. 28, no. 3, pp. 313-321, 2008.

[12] A. Sterodimas, J. de Faria, B. Nicaretta, and F. Boriani, "Autologous fat transplantation versus adipose-derived stem cellenriched lipografts: a study," Aesthetic Surgery Journal, vol. 31, no. 6, pp. 682-693, 2011.
[13] P. S. Nguyen, C. Desouches, A. M. Gay, A. Hautier, and G. Magalon, "Development of micro-injection as an innovative autologous fat graft technique: the use of adipose tissue as dermal filler," Journal of Plastic Reconstructive and Aesthetic Surgery, vol. 65, no. 12, pp. 1692-1699, 2012.

[14] B. Sommer and G. Sattler, "Current concepts of fat graft survival: histology of aspirated adipose tissue and review of the literature," Dermatologic Surgery, vol. 26, no. 12, pp. 1159-1166, 2000.

[15] E. Delay, S. Garson, G. Tousson, and R. Sinna, "Fat injection to the breast: technique, results, and indications based on 880 procedures over 10 years," Aesthetic Surgery Journal, vol. 29, no. 5, pp. 360-376, 2009.

[16] G. Rigotti, A. Marchi, P. Stringhini et al., "Determining the oncological risk of autologous lipoaspirate grafting for postmastectomy breast reconstruction," Aesthetic Plastic Surgery, vol. 34, no. 4, pp. 475-480, 2010.

[17] J. K. Fraser, M. H. Hedrick, and S. R. Cohen, "Breast surgery review article: oncologic risks of autologous fat grafting to the breast," Aesthetic Surgery Journal, vol. 31, no. 1, pp. 68-75, 2011.

[18] J. Y. Petit, E. Botteri, V. Lohsiriwat et al., "Locoregional recurrence risk after lipofilling in breast cancer patients," Annals of Oncology, vol. 23, no. 3, pp. 582-588, 2012.

[19] J. Y. Petit, V. Lohsiriwat, K. B. Clough et al., "The oncologic outcome and immediate surgical complications of lipofilling in breast cancer patients: a multicenter study-milan-paris-lyon experience of 646 lipofilling procedures," Plastic and Reconstructive Surgery, vol. 128, no. 2, pp. 341-346, 2011.

[20] J. Carvajal and J. H. Patiño, "Mammographic findings after breast augmentation with autologous," Aesthetic Surgery Journal, vol. 28, no. 2, pp. 153-162, 2008.

[21] M. A. Pelosi and M. A. Pelosi II, "Breast augmentation," Obstetrics and Gynecology Clinics of North America, vol. 37, no. 4, pp. 533-546, 2010.

[22] C.-F. Wang, Z. Zhou, Y.-J. Yan, D.-M. Zhao, F. Chen, and Q. Qiao, "Clinical analyses of clustered microcalcifications after autologous fat injection for breast augmentation," Plastic and Reconstructive Surgery, vol. 127, no. 4, pp. 1669-1673, 2011.

[23] C. Yi, Y. Pan, Y. Zhen et al., "Enhancement of viability of fat grafts in nude mice by endothelial progenitor cells," Dermatologic Surgery, vol. 32, no. 12, pp. 1437-1443, 2006.

[24] P. Butala, A. Hazen, C. Szpalski, S. M. Sultan, S. R. Coleman, and S. M. Warren, "Endogenous stem cell therapy enhances fat graft survival," Plastic and Reconstructive Surgery, vol. 130, no. 2, pp. 293-306, 2012.

[25] S. Hamed, O. Ben-Nun, D. Egozi et al., "Treating fat grafts with human endothelial progenitor cells promotes their vascularization and improves their survival in diabetes mellitus," Plastic and Reconstructive Surgery, vol. 130, no. 4, pp. 801-811, 2012.

[26] M. Jäger, P. Hernigou, C. Zilkens, M. Herten, J. Fischer, and R. Krauspe, "Cell therapy in bone healing disorders," Orthopedic Reviews, vol. 2, no. 2, pp. 79-87, 2010.

[27] M. F. Pittenger, A. M. Mackay, S. C. Beck et al., "Multilineage potential of adult human mesenchymal stem cells," Science, vol. 284, no. 5411, pp. 143-147, 1999.

[28] A. Alhadlaq and J. J. Mao, "Mesenchymal stem cells: isolation and therapeutics," Stem Cells and Development, vol. 13, no. 4, pp. 436-448, 2004.

[29] H. Castro-Malaspina, R. E. Gay, and G. Resnick, "Characterization of human bone marrow fibroblast colony-forming cells (CFU-F) and their progeny," Blood, vol. 56, no. 2, pp. 289-301, 1980. 
[30] D. J. Prockop, "Marrow stromal cells as stem cells for nonhematopoietic tissues," Science, vol. 276, no. 5309, pp. 71-74, 1997.

[31] M. F. Pittenger, "Mesenchymal stem cells from adult bone marrow," Methods in Molecular Biology, vol. 449, pp. 27-44, 2008.

[32] H. Kawaguchi, A. Hirachi, N. Hasegawa et al., "Enhancement of periodontal tissue regeneration by transplantation of bone marrow mesenchymal stem cells," Journal of Periodontology, vol. 75, no. 9, pp. 1281-1287, 2004.

[33] P. G. Robey and P. Bianco, "The use of adult stem cells in rebuilding the human face," Journal of the American Dental Association, vol. 137, no. 7, pp. 961-972, 2006.

[34] M. Jäger, E. M. Jelinek, K. M. Wess et al., "Bone marrow concentrate: a novel strategy for bone defect treatment," Current Stem Cell Research and Therapy, vol. 4, no. 1, pp. 34-43, 2009.

[35] Y. Wu, J. Wang, P. G. Scott, and E. E. Tredget, "Bone marrowderived stem cells in wound healing: a review," Wound Repair and Regeneration, vol. 15, no. 1, pp. S18-S26, 2007.

[36] P. K. Mishra, "Bone marrow-derived mesenchymal stem cells for treatment of heart failure: is it all paracrine actions and immunomodulation?" Journal of Cardiovascular Medicine, vol. 9, no. 2, pp. 122-128, 2008.

[37] S. H. Choi, S. Y. Jung, S. M. Kwon, and S. H. Baek, "Perspectives on stem cell therapy for cardiac regeneration. Advances and challenges," Circulation Journal, vol. 76, no. 6, pp. 1307-1312, 2012.

[38] D. Bulgin, E. Hodzic, and D. Komljenovic-Blitva, "Advanced and prospective technologies for potential use in craniofacial tissues regeneration by stem cells and growth factors," Journal of Craniofacial Surgery, vol. 22, no. 1, pp. 342-348, 2011.

[39] D. Bulgin, E. Irha, E. Hodzic, and B. Nemec, "Autologous bone marrow derived mononuclear cells combined with $\beta$-tricalcium phosphate and absorbable atelocollagen for a treatment of aneurysmal bone cyst of the humerus in child," Journal Biomaterials Applications, 2012.

[40] D. Bulgin and E. Hodzic, "Autologous bone marrow-derived mononuclear cells combined with $\beta$-TCP for maxillary bone augmentation in implantation procedures," The Journal of Craniofacial Surgery, vol. 23, no. 6, pp. 1728-3172, 2012.

[41] E. Tateishi-Yuyama, H. Matsubara, T. Murohara et al., "Therapeutic angiogenesis for patients with limb ischaemia by autologous transplantation of bone-marrow cells: a pilot study and a randomised controlled trial," Lancet, vol. 360, no. 9331, pp. 427435, 2002.

[42] J. P. Hunstad, "Tumescent and syringe liposculpture: a logical partnership," Aesthetic Plastic Surgery, vol. 19, no. 4, pp. 321-333, 1995.

[43] S. R. Coleman, "Structural fat grafting," Aesthetic Surgery Journal, vol. 18, no. 5, pp. 386-388, 1998.

[44] L. L. Q. Pu, S. R. Coleman, X. Cui, R. E. H. Ferguson, and H. C. Vasconez, "Autologous fat grafts harvested and refined by the coleman technique: a comparative study," Plastic and Reconstructive Surgery, vol. 122, no. 3, pp. 932-937, 2008.

[45] J. S. de Lezo, C. Herrera, M. Romero et al., "Functional improvement in patients with dilated cardiomyopathy after the intracoronary infusion of autologous bone marrow mononuclear cells," Revista Espanola de Cardiologia, vol. 66, no. 6, pp. 450-457, 2013.

[46] T. Asahara, T. Murohara, A. Sullivan et al., "Isolation of putative progenitor endothelial cells for angiogenesis," Science, vol. 275, no. 5302, pp. 964-967, 1997.
[47] K. C. Wollert and H. Drexler, "Clinical applications of stem cells for the heart," Circulation Research, vol. 96, no. 2, pp. 151-163, 2005.

[48] K. Yoshimura, K. Sato, N. Aoi, M. Kurita, T. Hirohi, and K. Harii, "Cell-assisted lipotransfer for cosmetic breast augmentation: supportive use of adipose-derived stem/stromal cells," Aesthetic Plastic Surgery, vol. 32, no. 1, pp. 48-55, 2008.

[49] J. H. Rosing, G. Wong, M. S. Wong, D. Sahar, T. R. Stevenson, and $\mathrm{L}$. L. Q. Pu, "Autologous fat grafting for primary breast augmentation: a systematic review," Aesthetic Plastic Surgery, vol. 35, no. 5, pp. 882-890, 2011.

[50] H. Wang, Y. Jiang, H. Meng, Q. Zhu, Q. Dai, and K. Qi, “Sonographic identification of complications of cosmetic augmentation with autologous fat obtained by liposuction," Annals of Plastic Surgery, vol. 64, no. 4, pp. 385-389, 2010. 


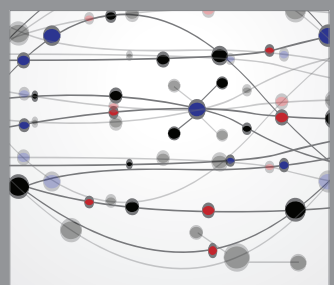

The Scientific World Journal
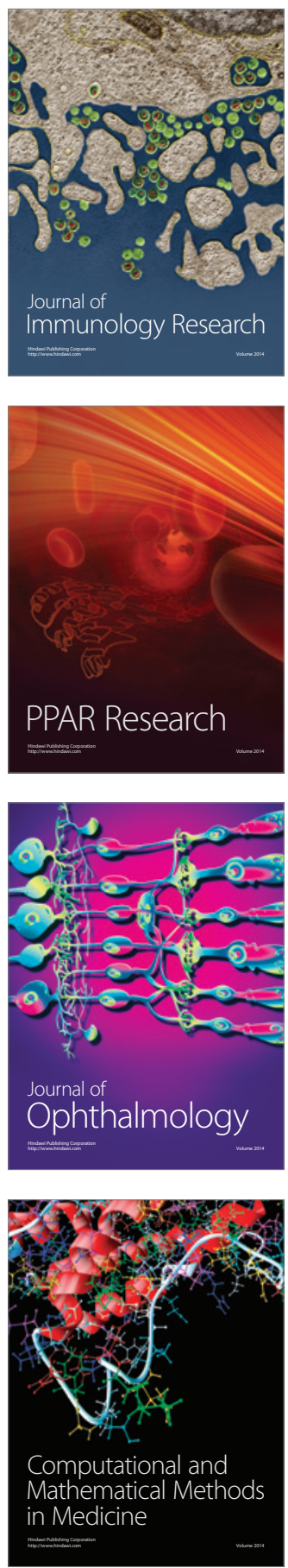

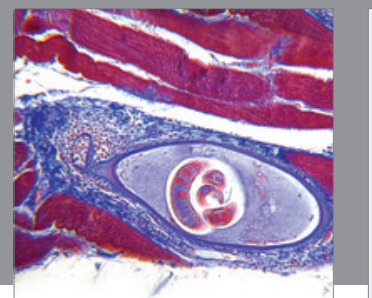

Gastroenterology

Research and Practice
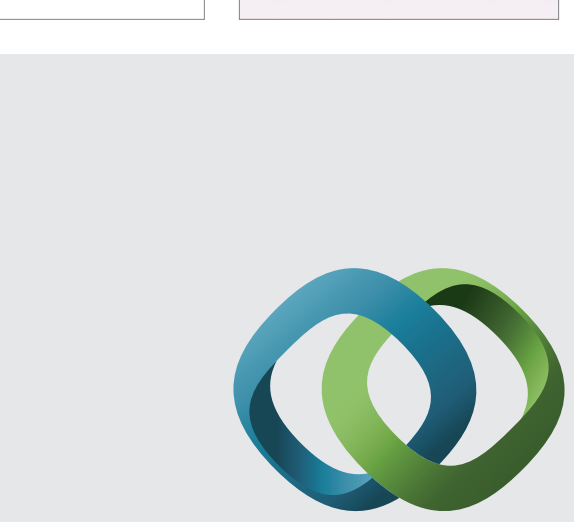

\section{Hindawi}

Submit your manuscripts at

http://www.hindawi.com
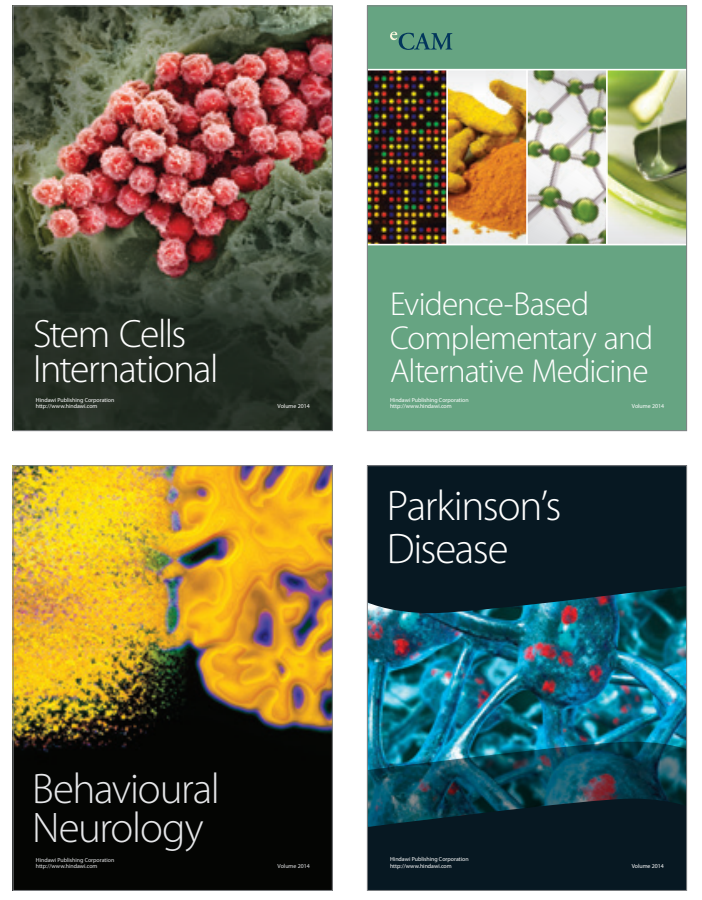
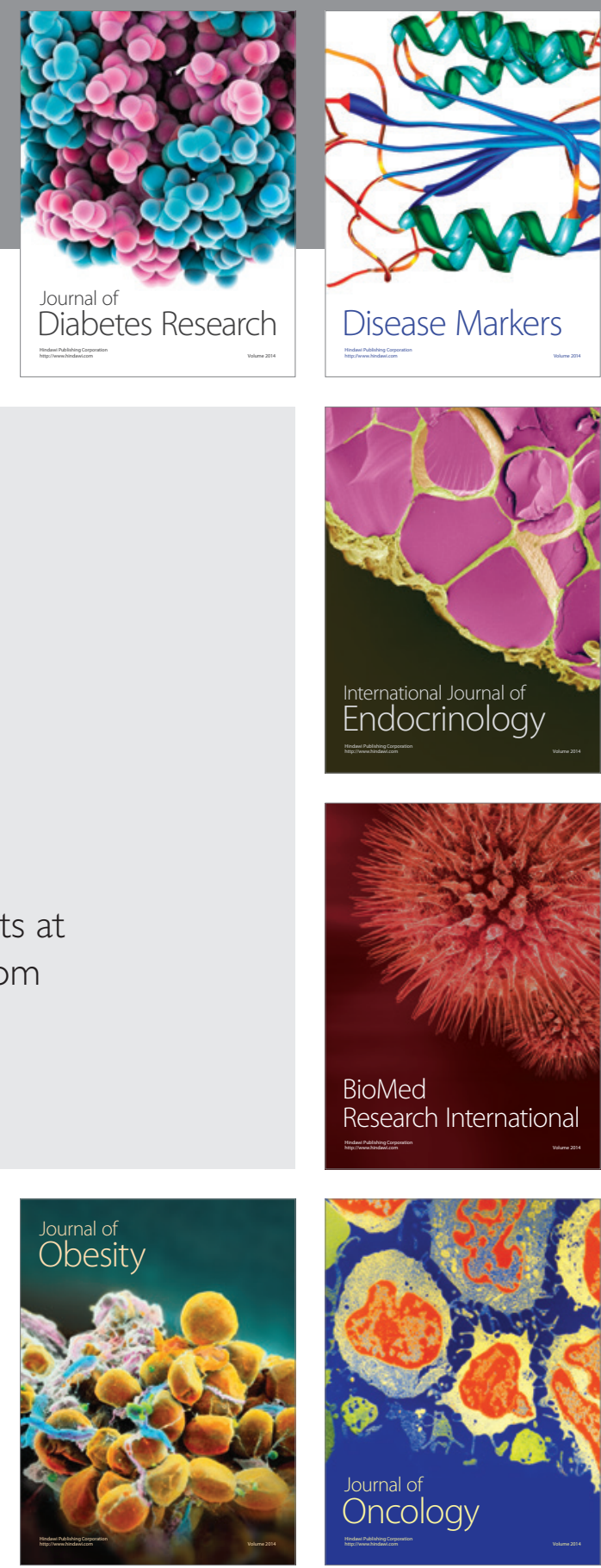

Disease Markers
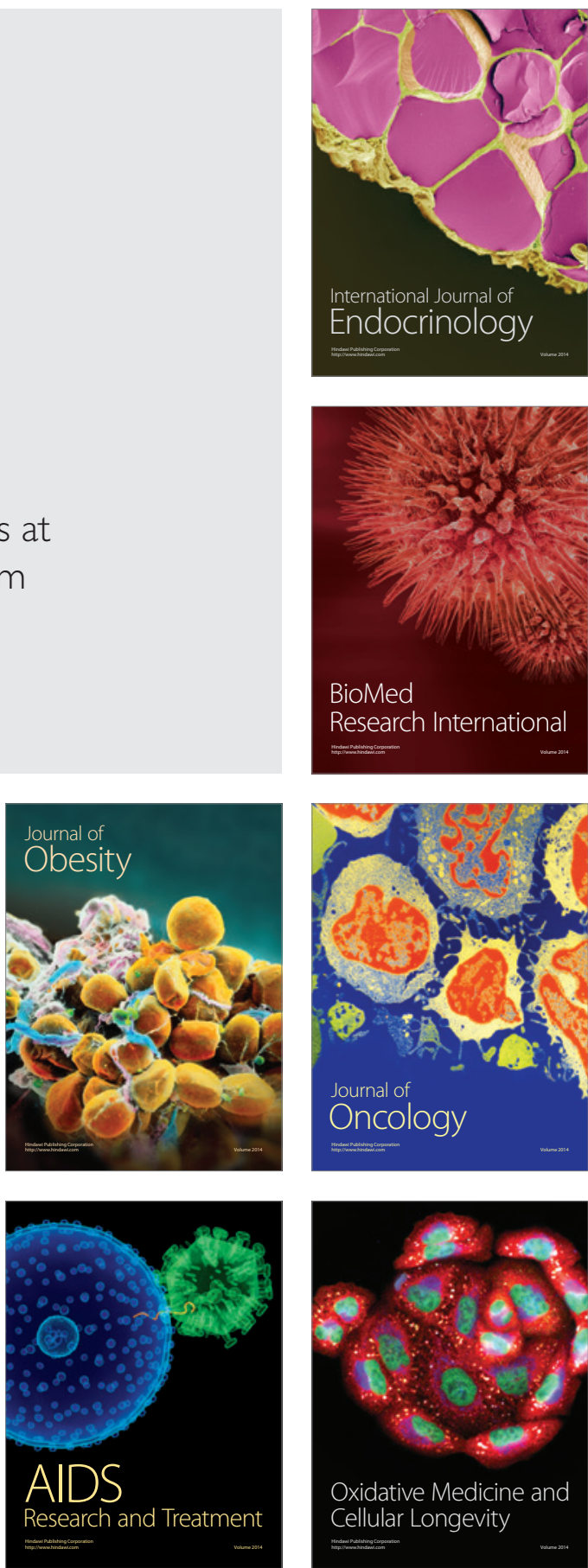